\title{
Micro-BLMs on highly ordered porous silicon substrates: rupture processes and lateral mobility
}

\author{
Daniela Weiskopf," Eva K. Schmitt," Marco H. Klühr, \\ Stephan K. Dertinger, ${ }^{\S}$ Claudia Steinem ${ }^{\#}$ * \\ Institut für Organische und Biomolekulare Chemie, Georg-August Universität, Tammannstr. 2, 37077 Göttingen, \\ Germany, Qimonda AG, Otto-Hahn-Ring 6, 81739 München, Germany
}

\section{- Supporting Information -}
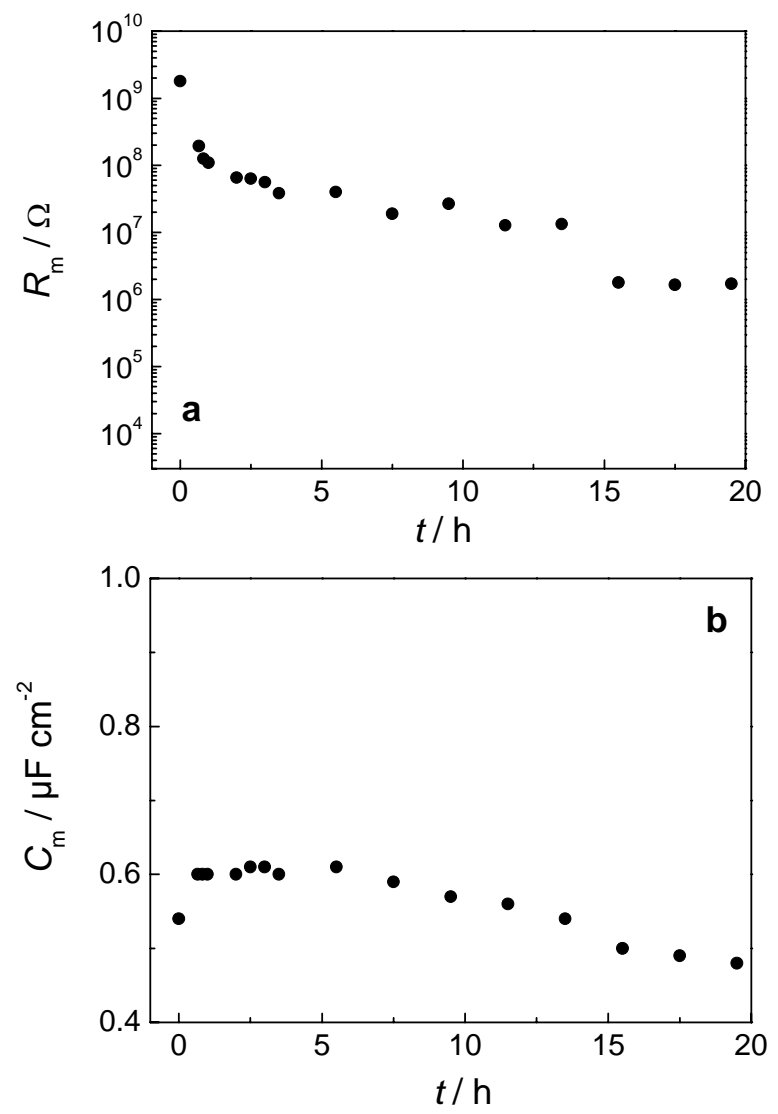

Figure S1. Change in membrane resistance (a) and specific membrane capacitance (b) as a function of time. The porous silicon substrate was functionalized with octadecanethiol (ODT). Micro-BLMs were prepared in the same was as those based on DPPTE-functionalized porous silicon substrates. The membrane resistance $R_{\mathrm{m}}$ and the specific membrane capacitance $C_{\mathrm{m}}$ were obtained from impedance spectra recorded in a frequency range of $10^{-2}-10^{6} \mathrm{~Hz}$. While the membrane resistance decreases over time, the membrane capacitance remains almost constant, which we attribute to residual amount of solvent that slightly changes the overall capacitance even if membranes suspending the pores have already been ruptured. 

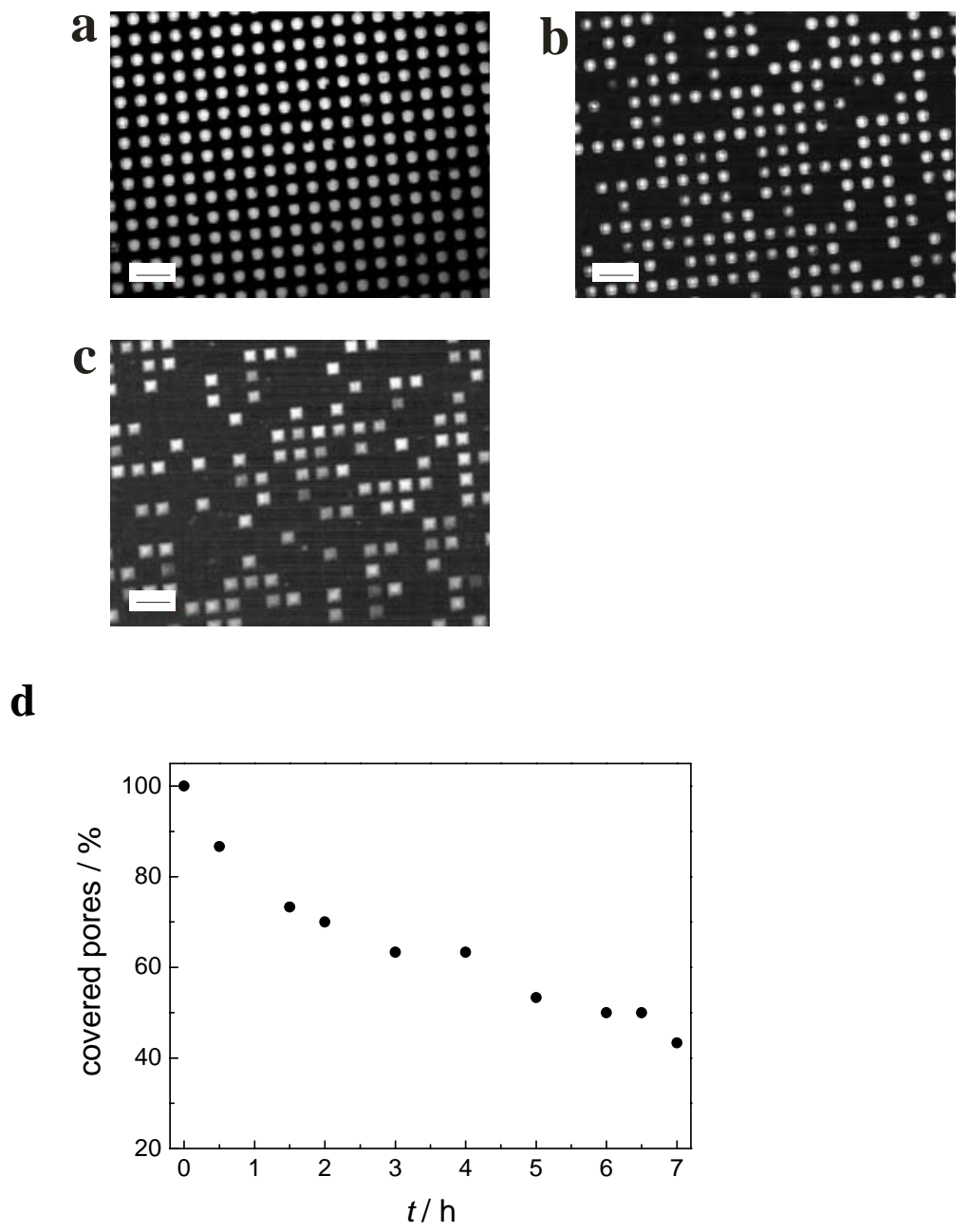

Figure S2. Fluorescence microscopy images of micro-BLMs generated on ODT-functionalized porous silicon substrates (a) $0 \mathrm{~h}$, (b) $3 \mathrm{~h}$ and (c) $7 \mathrm{~h}$ after its preparation. Micro-BLMs were obtained following the procedure for porous silicon substrates functionalized with DPPTE and doped with 0.1 mol\% $\beta$-BODIPY 500/510C12-HPC. The scale bar represents $20 \mu \mathrm{m}$. (d) Pixel analyses of the fluorescence images. The observed fluorescence at time $t=0 \mathrm{~h}$ was set to $100 \%$. 